\title{
Pronación consciente como parte del tratamiento de la neumonía por COVID-19
}

\author{
Recibido: 30/10/20 Aceptado: 26/11/20
}

Marcelo Zylberman¹, Carlos Ruiz², Cristina Estevan², Andrea Odzak , Florencia Arcondo', Leticia Fornasari ${ }^{1}$, Cristhian Armenteros ${ }^{1}$, Cecilia Garbarino ${ }^{1}$, Cecilia Brondo ${ }^{2}$, Noelia Sánchez Ejarque ${ }^{2}$, Daniel Nahmías ${ }^{2}$, Laura Fuertes ${ }^{2}$, Natalia Subat ${ }^{2}$, Marilin Medina², Facundo del Valle ${ }^{2}$, Jéssica Nieto $^{2}$, Analía Corradino², Julián Sahar ${ }^{3}$, Juan Gagliardi ${ }^{4}$.

\section{RESUMEN}

La pronación consciente es una de las herramientas utilizadas para reducir los ingresos a terapia intensiva (UTI) en la neumonía por COVID-19 con hipoxemia. Algunos pacientes no toleran estar en posición prono (intolerantes) y algunos que lo toleran no responden mejorando la saturación o su $\mathrm{PO}_{2}$. Presentamos una serie de 34 pacientes sometidos a pronación consciente; fueron tolerantes 18 (52,9\%). Nueve pacientes pasaron a UTI (26,4\%): 7 intolerantes $(43,7 \%)$ y 2 tolerantes $(11,1 \%)(p=0.038)$. No hallamos diferencias en la necesidad de ventilación mecánica y mortalidad entre tolerantes e intolerantes. De los 18 tolerantes se clasificó como respondedores a 10 pacientes $(55,5 \%)$. No hubo diferencia estadísticamente significativa en los pases a UTI entre los respondedores y no respondedores. La pronación consciente es una herramienta factible en el paciente con neumonía por COVID-19 y nos permitió predecir el requerimiento de terapia intensiva entre aquellos intolerantes al método.

Palabras clave: pronación consciente, neumonía, hipoxemia, COVID-19.
1 División Clínica Médica, Hospital Gral. de Agudos Dr. Cosme Argerich. GCBA. Buenos Aires, Argentina.

2 Unidad Kinesiología, Hospital Gral. de Agudos “Dr. Cosme Argerich. GCBA. Buenos Aires, Argentina.

${ }^{3}$ Residente de Clínica Médica, Hospital Gral. de Agudos Dr. Cosme Argeric". GCBA. Buenos Aires, Argentina.

${ }^{4}$ Comité de Etica en Investigación, Hospital Gral. de Agudos "Dr. Cosme Argerich. GCBA. Buenos Aires, Argentina.

Autor para correspondencia: Marcelo Zylberman. Olaya 1341, CABA, Argentina. CP 1414. Cel: 1144379117

Email:mzylberman@fibertel.com.ar

Estudio sin financiación. Los contenidos del material no han sido publicados anteriormente. Quienes figuran como autores participaron significativamente en la investigación o elaboración del manuscrito y se hacen responsables de todo su contenido. Ninguno presenta conflicto de intereses en relación a esta publicación. 


\section{Introducción}

La pandemia COVID-19 obligó a los equipos médicos a la búsqueda de recursos para responder a una demanda inesperada por su frecuencia y su gravedad.

Dentro del grupo interdisciplinario de trabajo de nuestro hospital nos propusimos que todo avance que pudiera evitar el pase a terapia intensiva (UTI) y a la necesidad de asistencia respiratoria mecánica (ARM) de los pacientes con neumonía debería ser explorado y utilizado en la medida que la bibliografía lo avalara.

Así, el uso de profilaxis antitrombótica, de hidroxicloroquina en la etapa inicial, la oxigenoterapia, la dexametasona y el plasma de convaleciente fueron discutidos y puestos a disposición para el objetivo trazado: colocar a la medicina interna como un muro de contención para evitar el desborde de camas de la terapia intensiva.

Colocar a los pacientes en posición de pronación es un recurso conocido en terapia intensiva, ya que en pacientes bajo ventilación mecánica mejora la oxigenación y reduce la mortalidad en formas severas del síndrome de distress respiratorio agudo (1).

La posición supina que adquieren los pacientes al ser internados puede ir en detrimento de la función pulmonar. Conlleva una sobreventilación de los alveolos ventrales e induce atelectasia de los alveolos posteriores. Esto favorece la compresión alveolar por la presión transmitida desde el abdomen por el diafragma. Existe también en posición supina un trastorno en la relación ventilación/ perfusión (mismatch $\mathrm{V} / \mathrm{Q}$ ) que dirige el flujo pulmonar a alveolos de las regiones posteroinferiores, pobremente ventiladas (2).

Rotar la posición de decúbito supino a prono supone una simple intervención para el paciente consciente internado en la sala, y podría mejorar la relación $\mathrm{V} / \mathrm{Q}$, reducir el shunt, favorecer el aclaramiento de secreciones y por consiguiente mejorar la función respiratoria.

Desde China, Europa y Estados Unidos Ilegó información acerca del beneficio de esta práctica en pacientes despiertos con COVID-19 al reducir los requerimiento de $\operatorname{ARM}(1,2)$.

Por tal motivo, nos propusimos evaluar los resultados de la implementación del prono consciente como alternativa no invasiva en nuestra sala de internación.

Nuestros objetivos fueron:

1. Conocer la frecuencia de tolerancia al prono de los pacientes con neumonía COVID-19.

2. Conocer la respuesta al prono de aquellos pacientes tolerantes.

3. Conocer si hay diferencias en la evolución (pase a UTI, ARM, mortalidad) entre aquellos pacientes tolerantes e intolerantes.

4. Conocer si hay diferencias en la evolución (pase a UTI, ARM, mortalidad) entre los respondedores y no respondedores.

\section{Materiales y métodos}

\section{Criterios de inclusión}

- Neumonía COVID-19 confirmada por PCR.

- Pacientes lúcidos, capaces de movilizarse por sí mismos y comunicarse sin dificultad.

- Estabilidad hemodinámica.

- $\quad$ Saturación de $\mathrm{O}_{2} \leq 94 \%$ al aire ambiente, medida por oximetría de pulso en posición supina al ingreso a la internación.

\section{Criterios de exclusión}

- Cirugía abdominal o torácica reciente (60 días).

- A todos los pacientes se les realizó una explicación verbal y mediante utilización de gráficas acerca de los cambios de decúbito de que debían adoptar, y solicitando permanezcan en posición prono, durante un período de 30 minutos a dos horas, antes de volver a la posición supina. Se solicitó realizar hasta cinco ciclos por día durante las horas de vigilia.

\section{Definiciones}

Tolerantes: aquellos pacientes capaces de permanecer por lo menos 30 minutos en la posición solicitada. 
Intolerantes: aquellos pacientes que no alcanzan a permanecer al menos 30 minutos en la posición solicitada.

Respondedores: aquellos con mejoría de la saturación de $\mathrm{O}_{2}$ en prono $\geq 3$ puntos porcentuales equivalente a 12 $\mathrm{mmHg}$ de $\mathrm{PO}_{2}$ en la saturación entre $90 \%$ o más, donde cada punto de saturación corresponde a $4 \mathrm{mmHg}$ (3).

Obesidad: índice de masa corporal (IMC) $>30$.

Se registraron las siguientes variables: datos demográficos, comorbilidades conocidas, saturación medida por oximetría de pulso y tolerancia.

Se registraron los datos de saturación con la misma fuente de oxígeno en posición supina (basal) y luego de 30 minutos en posición prono.

El modo de egreso se registró como: alta, pase a UTI, requerimiento de ARM y óbito.

\section{Análisis estadístico}

Las variables cualitativas se presentan como valores absolutos y su porcentaje y las variables cuantitativas como media \pm desvío estándar (DE) o mediana e intervalo intercuartilo (IIC) según el análisis de normalidad realizado mediante el test de Shapiro-Wilk. Para el análisis de las variables discretas se utilizó el test de Chi cuadrado (con corrección de Yates) o prueba exacta de Fisher. Para el análisis de los datos continuos se utilizó el test de $\mathrm{t} o$ test no paramétrico (Wilcoxon y Kruskall Wallis) según su distribución y el test de t para datos apareados para evaluar los cambios en la saturación con la pronación.

Se utilizó para el análisis el programa Epilnfo versión 7.2.4 y Stata/SE® 13.0 para Windows. Se consideró significativo un valor $\mathrm{p}<0.05$.

\section{Resultados}

Entre el 10 de mayo y el 31 de julio de 2020 se incluyeron 34 pacientes (p). El promedio de edad fue 53,9 $\pm 9,6$ años (34-68). Veinte fueron hombres $(58,8 \%)$ y 14 mujeres.

Tenían comorbilidades previas 21 pacientes $(61,8 \%)$ : de ellos, doce eran obesos (35,2\%), seis diabéticos $(17,6 \%)$, seis hipertensos (17,6\%), dos con cáncer, uno con asma y uno con hipertensión pulmonar.

Siete pacientes $(20,5 \%)$ tenían más de una comorbilidad.

Al ingreso al estudio, 27 pacientes se hallaban respirando aire ambiente, tres con cánula nasal y cuatro con máscara reservorio.

La mediana de saturación al ingreso fue de $91 \%$ en los que respiraban aire ambiente, de 95,5\% entre los que respiraban con cánula nasal y de 95,5\% entre los que usaban máscara reservorio. La mediana de saturación basal en supino fue $93 \%$ y al pasar al prono de $96 \%(p<0.001)$ en el global de la población (Tabla 1).

Fueron tolerantes 18 de los 34 pacientes (52,9\%). De ellos, dos pasaron a UTI $(11,1 \%)$.

De estos 18 tolerantes se clasificó como respondedores a 10 pacientes (55,5\%); uno de ellos pasó a UTI, no requirió ARM y fue dado de alta. Fueron no respondedores 8 (44,5\%); uno de ellos pasó a UTI (12,5\%), requirió ARM y falleció.

Fueron intolerantes 16 pacientes (47,1\%). Entre ellos, hubo siete pases a UTI (43,7\%); cinco de ellos requirieron ARM $(31,2 \%)$ y hubo tres óbitos $(18,7 \%)$ (Tabla 2$)$.

Por lo tanto, hubo nueve pases a UTI $(26,4 \%)$ : siete de los

\section{Tabla 1. Saturación al ingreso a la internación, basal pre y post prono}

\begin{tabular}{|l|c|c|c|c|}
\multicolumn{1}{|c|}{ Saturación inicial } & $\mathbf{n}$ & Mediana & IIC & Rango \\
\hline Global & 34 & 91 & $90-93$ & $75-98$ \\
\hline $21 \%$ & 28 & 91 & $88,5-92,5$ & $75-95$ \\
\hline Cánula & 2 & 95,5 & $93-98$ & $93-98$ \\
\hline Reservorio & 4 & 95,5 & $92-96$ & $89-96$ \\
\hline Sat. pre y post prono global & Md & IIC & Rango & \\
\hline Basal supino & 93 & $91-94$ & 8998 & dif 2,66; $p<0,001$ (test de t para datos apareados) \\
\hline Prono & 96 & $93-98$ & $91-99$ & .
\end{tabular}




\begin{tabular}{|c|c|c|c|c|c|}
\hline & \multicolumn{2}{|c|}{ Tolerantes } & \multicolumn{2}{|c|}{ Intolerantes } & \multirow[t]{2}{*}{ p } \\
\hline & $\mathrm{n}$ & $\%$ o IIC & $\mathbf{n}$ & $\%$ o IIC & \\
\hline $\mathrm{N}$ & 18 & 52,9 & 16 & 47,1 & 0,18 \\
\hline Sexo masc & 13 & 72,2 & 8 & 50,0 & 0,18 \\
\hline Edad & \multicolumn{2}{|c|}{$53,2 \pm 8,4$} & \multicolumn{2}{|c|}{$54,9 \pm 10,9$} & 0,60 \\
\hline Obesidad & 6 & 33,3 & 6 & 37,5 & 0,79 \\
\hline HTA & 5 & 27,8 & 1 & 6,3 & 0,10 \\
\hline DBT & 3 & 16,7 & 3 & 18,8 & 0,87 \\
\hline \multicolumn{6}{|c|}{ Saturación al ingreso } \\
\hline $21 \%$ & 91 & $88-91,5$ & 91 & $89,5-93$ & 0,60 \\
\hline Cánula & 95 & $93-96$ & 97 & $94,5-97$ & 0,23 \\
\hline Reservorio & 95 & $93-97$ & 96 & $95-98$ & 0,24 \\
\hline \multicolumn{6}{|l|}{ PRONO } \\
\hline Pre-prono & 93 & $91-94$ & & & \\
\hline En-prono & 96 & $93-98$ & & & \\
\hline UTI & 2 & 11,1 & 7 & 43,7 & 0,038 \\
\hline ARM & 1 & 50 & 5 & 71,4 & 0,58 \\
\hline Óbito & 1 & 5,6 & 3 & 18,7 & 0,25 \\
\hline
\end{tabular}

intolerantes y dos de los tolerantes $(p=0.038)$. No hallamos diferencias en la necesidad de ARM y mortalidad entre tolerantes e intolerantes (Tabla 2).

Seis de los 18 pacientes tolerantes no alcanzaron hasta 95\% de saturación en prono. Cinco de ellos fueron dados de alta y uno pasó a UTI, requirió ARM y falleció.

No se observó relación significativa entre la presencia de factores de riesgo como hipertensión, obesidad, diabetes y la respuesta al prono o la evolución.

La media de la edad de los dados de alta fue $52.96 \pm 9.6$ años y de los óbitos fue $61.5 \pm 5.7(p=0.09)$

De los cuatro óbitos, uno tenía obesidad y diabetes, uno obesidad, uno cáncer y uno no tenía comorbilidades Tres de ellos fue entre los intolerantes y uno entre los tolerantes.

Entre los tolerantes respondedores y no respondedores no hubo diferencia estadísticamente significativa para pase a UTI (Tabla 3).

\section{Discusión}

Evitar un ingreso a terapia intensiva en la pandemia requiere de múltiples herramientas a utilizar en forma simultánea o sucesiva. Nuestro grupo muestra en esta serie que el modo de pronación consciente puede ser útil para evaluar el riesgo de pase a UTI de los pacientes con neumonía COVID. Sun y col. publicaron su experiencia en la provincia de Jiamgsu (China) por la cual a través de la pronacion consciente lograron bajar del 10 al $1 \%$ la cantidad de pacientes que requirieron ventilación mecánica (4), y otros grupos, como los de Caputo en New York (5) y Thompson en Columbia (6), sumaron su experiencia en este método. Estos resultados alentaron al Instituto Nacional de la Salud de Estados Unidos $(\mathrm{NIH}$, por sus siglas en inglés) a recomendar en sus guías, si bien con clase IIIC, el uso de una prueba de prono consciente en pacientes que no estén en riesgo de intubación inminente (7).

El estudio de Caputo y col. sorprendió al comunicar que sus 50 pacientes con saturación de $84 \%$ al aire en posición supina, en cinco minutos en prono pasaban a saturar $94 \%$. El $24 \%$ de ellos requirió intubación dentro de las 24 hs, pero de los otros 37 pacientes que continuaron con el prono, solo cinco fueron ventilados (5).

En Columbia, Thompson y col. vieron en sus 25 pacientes que cuando la saturación en prono ascendia a $95 \%$ o más, el $37 \%$ requería pase a UTI y ventilación mecánica, pero cuando la saturación no alcanzaba $95 \%$ en prono, el $83 \%$ de los casos se ventilaba (6). Lo interesante de este estudio es que de los 25 casos, 13 fueron dados de alta sin ingresar a ventilación 


\section{Tabla 3. Diferencias entre los respondedores y no respondedores}

\begin{tabular}{|c|c|c|c|c|c|}
\hline & \multicolumn{2}{|c|}{ Respondedores } & \multicolumn{2}{|c|}{ No Respondedores } & \multirow[t]{2}{*}{$\mathbf{p}$} \\
\hline & $\mathbf{n}$ & $\%$ & $\mathbf{n}$ & $\%$ & \\
\hline $\mathrm{N}$ & 10 & 55,6 & 8 & 44,4 & \\
\hline Sexo masc & 7 & 79 & 6 & 75 & 0,61 \\
\hline Edad & \multicolumn{2}{|c|}{$54,3 \pm 7$} & \multicolumn{2}{|c|}{$51,7 \pm 10,3$} & 0,54 \\
\hline Obesidad & 3 & 30 & 3 & 37,5 & 0,56 \\
\hline HTA & 4 & 40 & 1 & 12,5 & 0,22 \\
\hline DBT & 3 & 30 & 0 & & 0,14 \\
\hline \multicolumn{6}{|c|}{ Saturación al ingreso } \\
\hline $21 \%$ & 90,5 & $88-91$ & 91 & $90-93$ & 0,27 \\
\hline Cánula & 94 & $92-94$ & 96 & $95-98$ & 0,03 \\
\hline Reservorio & 94,5 & $93-97$ & 96 & $89-97$ & 1,00 \\
\hline \multicolumn{6}{|l|}{ PRONO } \\
\hline Pre-prono & 93 & $91-94$ & 93 & $91-94,5$ & 0,96 \\
\hline En-prono & 97 & $96-98$ & 93 & $91-96,5$ & 0,06 \\
\hline T test apareado & \multicolumn{2}{|c|}{ dif 4,10; $p<0,0001$} & \multicolumn{2}{|c|}{ dif 0,$87 ; p=, 041$} & \\
\hline UTI & 1 & & 1 & & \\
\hline ARM & 0 & & 1 & & \\
\hline Óbito & 0 & & 1 & & \\
\hline
\end{tabular}

mecánica, y de ellos 12 fueron los que alcanzaron saturaciones de $95 \%$ o más en prono. En nuestra serie, seis pacientes no llegaron a saturar $95 \%$ en prono, pero solo uno de ellos fue a UTI, requirió ARM y falleció.

Nuestro grupo aceptó como respondedor a aquel paciente con ascenso de 3 puntos porcentuales de saturación, lo cual corresponde a un ascenso de la $\mathrm{PO}_{2}$ de $12 \mathrm{mmHg}$ (3), similar al $20 \%$ de ascenso de la $\mathrm{PO}_{2}$ que exige el grupo de Elharrar y colaboradores (8).

En el estudio de Elharrar, cuatro (16,6\%) de sus 24 casos fueron intolerantes, cinco toleraron entre una y tres horas, y $15(63 \%)$ toleraron el prono más de tres horas. El grupo italiano (1) publica una cifra mucho más alta de tolerantes (83,9\%). En nuestra serie, el $47 \%$ de los pacientes fueron intolerantes: si bien seis de ellos eran obesos, hubo la misma cantidad de obesos en el grupo de los tolerantes.

Dentro de los tolerantes, Elharrar divide en respondedores persistentes y parciales (8). De su grupo de 15 respondedores, solo tres fueron persistentes. Para el grupo italiano, si bien el $50 \%$ era respondedor, al volver a supinarlos la $\mathrm{Pa} / / \mathrm{Fi}$ (cociente entre la presión parcial de oxígeno/fracción inspirada de oxígeno) se asemejaba al valor pre pronación (1). No medimos en nuestra serie a respondedores parciales y persistentes.

En una editorial de JAMA (9) se acepta una tasa de éxito del prono consciente del $25 \%$ entre los respondedores. No seaclara si los respondedores persistentes tienen mejor pronóstico que los respondedores parciales. El pase a UTI en nuestra serie fue claramente superior en los pacientes intolerantes, similar a lo que demostraran Caputo y colaboradores (5), pero no hubo diferencias en pase a UTI entre respondedores y no respondedores. Esto se asemeja a lo publicado por el grupo italiano, donde no hubo diferencias entre los respondedores y no respondedores en el $28 \%$ de sus pacientes que requirieron $\operatorname{ARM}(1)$.

Aunque incierta hasta que aparezcan estudios randomizados, parece ser que la pronación consciente ha venido a ocupar un lugar en el manejo de la insuficiencia respiratoria en vistas al futuro (10).

Nuestro estudio, limitado tanto por el número de pacientes y por ser observacional y sin grupo control, demuestra que la pronación consciente es una herramienta factible en el manejo del paciente con neumonía por COVID-19 y colabora en la predicción de requerimientos de UTI entre aquellos pacientes intolerantes al método. 


\section{Bibliografía}

1. Coppo A, Bellani G, Winterton D, Di Pierro M, Soria A, Faverio P. et al. Feasibility an physiological effects of prone positioning in non-intubated patients with acute respiratory failure due to COVID-19: a prospective cohort study. Lancet Respir Med 2020; 8: 765-74.

2. Jiang L, LeBaron J, Bodnar D, Caputo N, Chang B, Chiricolo G, et al. Conscious Proning: An Introduction of Proning Protocol for Nonintubated, Awake, Hypoxic Emergency Department COVID-19 Patients. Acad Emerg Med 2020; 27: 566-9.

3. Madan A. Correlation between the levels of SpO2and PaO2. Lung India. 2017;34(3):307-308. doi:10.4103/ lungindia.lungindia_106_17

4. Sun Q, Qiu $H$, Huang $M$, Yang $Y$. Lower mortality of COVID-19 by early recognition and intervention: experience from Jiangsu Province. Ann Intensive Care 2020; 10:33 https://doi.org/10.1186/s13613020-00650-2

5. Caputo N, Strayer R, Levitan R. Early Self-Proning in Awake, Non-intubated Patients in the Emergency Department: A Single ED's Experience During the COVID-19 Pandemic. Acad Emerg Med 2020; 27:3758

6. Thompson A, Ranard B, Wei $\mathrm{Y}$, Jelic S. Prone Positioning in Awake, Nonintubated Patients With COVID-19 Hypoxemic Respiratory Failure. JAMA Intern Med. Published Online June 17, 2020. doi:10.1001/ jamainternmed.2020.3030

7. COVID-19 Treatment Guidelines Panel. Coronavirus Disease 2019 (COVID-19) Treatment Guidelines. National Institutes of Health. Available at https://www. covid19treatmentguidelines.nih.gov/. Accessed July, 17

8. Elharrar $X$, Trigui $Y$, Dols A-M, Touchon F, Martinez $S$, Prud'homme $E$, et al. Use of Prone Positioning in Nonintubated Patients With COVID-19 and Hypoxemic Acute Respiratory Failure. JAMA 2020;323:2336-8

9. Sarma A, Calfee C. Prone Positioning in Awake, Nonintubated Patients With COVID-19: Necessity Is the Mother of Invention. JAMA Intern Med. Published Online: June 17, 2020. doi:10.1001/ jamainternmed.2020.3027

10. Telias I, Katira B, Brochard L. Is the Prone Position Helpful During Spontaneous Breathing in Patients With COVID-19? JAMA 2020; 323: 2265-7. 
Prone positioning in awake patients as part of treatment in patients with COVID-19 pneumonia

The prone positioning (PP) in awake patients is one of the tools to reduce the number of admissions to Intensive Care Unit (ICU) in cases of Covid-19 hipoxemic pneumonia. Some patients do not tolerate PP (intolerants) and others that tolerate it do not respond with improvement of $\mathrm{PO} 2$ or oxygen saturation. We present here a series of 34 patients who underwent PP. Eighteen of them tolerated PP (52,9\%). Nine patients $(26,4 \%)$ were admitted to ICU: 7 who had not tolerated PP $(43,7 \%)$ and 2 who had tolerated PP $(11,1 \%)(p=$ 0.038 ). We did not find differences in the need for mechanical ventilation and mortality between patients who tolerated and who did not tolerate PP. From those 18 who tolerated PP, 10 were classified as responders (55,5\%). We did not find any significant statistical differences for admission to ICU between responders and non-responders. PP in awake patients is a feasible tool in cases of COVID-19 Pneumonia, and it allowed us to predict the requirements of ICU between those who were not tolerant to the method.

Keywords: prone, awake, pneumonia, hypoxemia, COVID-19. 